\title{
Relação de citocinas inflamatórias e PCR e risco cardiovascular em pacientes com doença de Crohn
}

\author{
Relationship inflammatory cytokine and CRP and cardiovascular risk in patients with Crohn's \\ disease
}

Relación de citocinas inflamatorias y PCR y riesgo cardiovascular en pacientes con enfermedad de

Crohn

Thaline Milany da Silva Dias

ORCID: https://orcid.org/0000-0003-1734-4312 Universidade Federal do Piauí, Brasil E-mail: thatadias77@gmail.com

Bruna Emanuele Pereira Cardoso ORCID: https://orcid.org/0000-0002-3863-1276 Universidade Federal do Piauí, Brasil E-mail: brunaemanuelec@hotmail.com

Ayres Fran da Silva e Silva

ORCID: https://orcid.org/0000-0001-7954-1368 Universidade Federal do Piauí, Brasil E-mail: ayressilvaesilva@gmail.com

Rafaelly Raiane Soares da Silva ORCID: https://orcid.org/0000-0002-0049-3162 Universidade Federal do Piauí, Brasil E-mail: rafaelly.nut@gmail.com

Taynáh Emannuelle Coelho de Freitas ORCID: https://orcid.org/0000-0003-3459-6933 Universidade Federal do Piauí, Brasil E-mail: taynahmanu@gmail.com Juliana Soares Severo

ORCID: https://orcid.org/0000-0002-1771-7871 Universidade Federal do Piauí, Brasil E-mail: ju_ssevero@hotmail.com

Guida Graziela Santos Cardoso ORCID: https://orcid.org/0000-0002-8639-7146 Universidade Federal do Piauí, Brasil E-mail: guida.cardoso18@gmail.com

Letícia de Sousa Milanez

ORCID: https://orcid.org/0000-0002-9890-7481 Universidade Estadual do Piauí, Brasil E-mail: leticia-sousa123@hotmail.com

Alana Rafaela da Silva Moura ORCID: https://orcid.org/0000-0002-4089-8623 Universidade Federal do Piauí, Brasil E-mail: alanarafaelasmoura@gmail.com

Jennifer Beatriz Silva Morais

ORCID: https://orcid.org/0000-0002-9055-7851 Universidade Federal do Piauí, Brasil

E-mail: jenniferbeatriz.morais@gmail.com

\begin{abstract}
Resumo
Objetivo: Investigar e correlacionar o nível de PCRus e citocinas pró-inflamatórias como sinalizadores de inflamação e verificar o risco cardiovascular nos pacientes com doença de Crohn. Métodos: O estudo foi composto por 36 pacientes (18 controle e 18 caso) Resultados: As análises mostraram níveis séricos de PCRus, IL-10, IFN- $\gamma$, IL-8, TNF- $\alpha$ maiores nos pacientes com a doença quando comparados ao grupo controle, com diferença significativa em relação à PCRus. Observou-se que a média de 131,12, colesterol total, 40,85, HDL-c e 76,05, LDL-c para o grupo caso Conclusões: Níveis de PCRus e citocinas mais elevados no grupo caso sugere que os pacientes com doença de Crohn apresentam risco cardiovascular aumentado quando comparados com pessoas sem a doença e com outros fatores de risco.
\end{abstract}

Palavras-chave: Inflamação; Doença de Crohn; Doenças cardiovasculares; Proteína C-Reativa. 


\begin{abstract}
Objective: To investigate and correlate the level of CRP and pro-inflammatory cytokines as signs of inflammation and to verify cardiovascular risk in patients with Crohn's disease. Methods: The study was composed of 36 patients (18 control and 18 case) Results: The analyzes showed serum levels of CRP, IL-10, IFN- $\gamma$, IL-8, TNF- $\alpha$ higher in patients with the disease when compared to control group, with significant difference in relation to CRP. It was observed that the mean of 131.12, total cholesterol, 40.85, HDL-c and 76.05, LDL-c for the case group Conclusions: Higher CRP and cytokine levels in the case group suggests that patients with Crohn's disease has increased cardiovascular risk when compared to people without the disease and other risk factors.
\end{abstract}

Keywords: Inflammation; Crohn disease; Cardiovascular diseases; C-Reactive protein.

\title{
Resumen
}

Objetivo: Investigar y correlacionar el nivel de PCR y citocinas proinflamatorias como signos de inflamación y verificar el riesgo cardiovascular en pacientes con enfermedad de Crohn. Métodos: El estudio estuvo compuesto por 36 pacientes (18 controles y 18 casos) Resultados: Los análisis mostraron niveles séricos de PCR, IL-10, IFN- $\gamma$, IL-8, TNF- $\alpha$ más altos en pacientes con la enfermedad en comparación con grupo control, con diferencia significativa en relación a la PCR. Se observó que la media de 131,12, colesterol total, 40,85, HDL-c y 76,05, LDL-c para el grupo de casos Conclusiones: Los niveles más altos de PCR y citocinas en el grupo de casos sugieren que los pacientes con enfermedad de Crohn tienen un mayor riesgo cardiovascular en comparación a personas sin la enfermedad y otros factores de riesgo.

Palabras clave: Inflamación; Enfermedad de Crohn; Enfermedades cardiovasculares; Proteína C-reactiva.

\section{Introdução}

A doença de Crohn (DC) é uma afecção de natureza sistêmica que pode afetar qualquer segmento do sistema digestório (Soares; Schauren; Stroparo, 2018), descrita como uma condição inflamatória crônica, resultante de ativação persistente e inadequada do sistema imune mucoso. Essa desregulação do sistema imune manifesta-se através de uma elevação local de diversos mediadores inflamatórios, como as citocinas e a proteína C reativa (PCR) (Papacosta et al., 2017).

A proteína C reativa (PCR) é uma proteína de fase aguda e um marcador inflamatório. Antigamente esta proteína era vista somente como um determinante de inflamação vascular, entretanto, atualmente sabe-se que, quando dosada por meio de técnicas de alta sensibilidade seus níveis podem ser relacionados a eventos cardíacos futuros, até mesmo em pacientes com resultados normais de troponina T e I. As técnicas para dosagem de proteína C reativa ultrassensível (PCR-us) detectam níveis muito baixos dessa proteína que desempenha função ativa na aterosclerose, o que demonstra seu valor como biomarcador de risco cardiovascular (Ribas et al., 2015).

Cabe destacar que a PCR é produzida por hepatócitos em níveis reduzidos em circunstâncias normais, porém em condições de inflamação sistêmica sua concentração sobe rapidamente sob a influência de citocinas pró-inflamatórias, incluindo a interleucina-6 (IL-6) (Valadasa et al., 2019) e fator de necrose tumoral alfa (TNF- $\alpha$ ). Essa proteína apresenta forte correlação com marcadores de atividade clínica, endoscópica, radiológicas e transversais nas desordens metabólicas, especialmente na DC. Além disso, exerce a vantagem de um aumento precoce após o início da inflamação e uma diminuição rápida após a sua resolução, devido à sua meia-vida curta de 19 h (Vilela et al., 2012).

A expressão elevada de PCR está associada em duas a três vezes com a prevalência de infarto de miocárdio e doença vascular periférica e à previsão de incidência de eventos cardiovasculares, sendo mais eficiente, por exemplo, que os níveis de LDL nos pacientes com ou sem doença cardiovascular pré-existente (Kalogeropoulos et al., 2014; Koenig, 2013; Sercelik et al., 2021). Além disso, foi demonstrada a correlação entre as citocinas e a determinação por endoscopia da inflamação em segmentos do intestino, o que sugere o papel potencial destes marcadores para determinar a atividade da doença de Crohn (Vilela et al., 2012).

A DC pode ser resultado de uma falha primária na regulação dos linfócitos e citocinas, como a interleucina-10 (IL-10) e fator $\beta$ de necrose tumoral (TNF- $\beta$ ), em controlar a inflamação e alvos efetores (Ferraz, 2016; Ferreira et al., 2010). A mucosa torna-se susceptível à ação da prostaglandina E2 (PGE2), leucotrieno B4, IL-8, IL-1, IL-10, IL-12, interferon gama 
(INF- $\gamma$ ) e mais recentemente tem sido descrito a IL-17 (Torres et al., 2011) ou seja, anormalidades típicas dos processos inflamatórios mediados pelos linfócitos auxiliares T1 (Th1) (Ferraz, 2016; Ferreira et al., 2010).

$\mathrm{Na}$ mucosa inflamada da DC há maior expressão de citocinas pró-inflamatórias (IL-1, IL-6, IL-8, TNF- $\alpha$ ), quimiocinas e vários mediadores lipídicos. O desequilíbrio entre citocinas pró e anti-inflamatórias condicionará a intensidade e duração da resposta inflamatória (Ferreira et al., 2010). Assim, o objetivo do presente estudo foi investigar e correlacionar o nível de PCRus e citocinas pró-inflamatórias como sinalizadores de inflamação e verificar o risco cardiovascular nos pacientes com doença de Crohn.

\section{Metodologia}

\section{Protocolo Experimental}

Trata-se de um estudo transversal caso-controle, de natureza quantitativa e analítica, desenvolvido no ambulatório de Doenças Inflamatório Intestinal sediado no Hospital Universitário da Universidade Federal do Piauí (HU-UFPI). A população do estudo foi composta por pacientes diagnosticados com doença de Crohn em tratamento no referido hospital. O grupo controle foi composto de profissionais da limpeza da UFPI e, de operários da construção civil, selecionados ao acaso. O estudo contou com 36 pacientes (18 grupo controle e 18 grupo caso).

A pesquisa utilizou como critério de exclusão: os menores de 18 anos, pessoas vulneráveis (presidiários, índios, pessoas com capacidade mental ou autonomia reduzida), pacientes diagnosticados com retocolite ulcerativa ou colite indeterminada para o grupo caso e para o grupo controle aqueles que referissem à presença de doença inflamatória, os que usavam anti-inflamatório no momento da coleta ou nos últimos cinco dias e os encontrava com febre no momento da coleta ou tinha tido febre nos últimos dias.

Os exames físicos e os dados de identificação e socioeconômicos foram coletados através de questionário previamente elaborado. Para determinar o perfil lipídio e concentração de citocina realizou punção de sangue venoso dos participantes. Coletou-se ainda com a autorização da comissão de ética do hospital os resultados bioquímicos contidos nos prontuários dos participantes com doença de Crohn. Para o estado nutricional global realizou a medida do Índice de Massa Corpórea (IMC), para tanto, aferiu as medidas de peso, através de uma balança digital e à estatura pelo estadiômetro com haste móvel.

Para avaliar a gravidade da doença de Crohn, adotou o Índice de Atividade da doença de Crohn (IADC) para diferenciá-la em ativa ou remissão. IADC < 150 indica doença em remissão, enquanto que o IADC > 150 indica que a doença está ativa. Entre 150 e 250 indica estado leve; IADC entre 250 e 350, estado moderado e maior que 350, grave8.

As amostras para dosagem de Citocinas e PCRus

Na coleta da amostra de sangue usou dois tubos de análise a vácuo contendo gel separador, em um dos tubos coletou uma alíquota de $10 \mathrm{~mL}$ e no outro $5 \mathrm{~mL}$ de sangue por punção venosa dos participantes, após 12 horas de jejum. E, em seguida os tubos foram levados para o laboratório de Bioquímica da UFPI e centrifugadas (Fanem) por 5 minutos a uma velocidade de $5000 \mathrm{rpm}$ e obtido o soro. Um dos tubos a vácuo o soro foi transferido para um par de eppendorf identificados e armazenados em refrigerador a -800C, para posterior análise de citocinas e PCRus. O soro do outro tubo foi transferido para um eppendorf e imediatamente utilizado nas análises do perfil lipídeos.

\section{Níveis de Citocinas e PCRus}

No Laboratório de Imunologia do Departamento de Análises Clínicas, Toxicológicas e Bromatológicas da Faculdade de Ciências Farmacêuticas de Ribeirão Preto (USP) determinou nos níveis séricos das interleucinas: IL-10, IL-8, TNF- $\alpha$, IFN- $\gamma$ pela técnica de ELISA e de acordo com as instruções do fabricante do kit comerciais da R\&D Systems® (Minneapolis, MN, 
EUA) e os níveis de PCRus pela metodologia de imunoturbidimetria do Kit Multicontrol Bioclin®.

\section{Perfil Lipídico}

No Laboratório de Pesquisa do Setor de Bioquímica da Universidade Federal do Piaúi realizou as dosagens do colesterol total, o HDL-colesterol e os triglicerídeos pelo método colorimétrico enzimático do fabricante (Labtest $\left.{ }^{\circledR}\right)$. E, para determinar os níveis do LDL-colesterol aplicou a fórmula proposta por Friedwald, na qual LDL-colesterol = CT - (HDLcolesterol + TG/5).

\section{Aspectos Éticos}

O projeto de pesquisa foi aprovado pelo Comitê de Ética em Pesquisa da UFPI - CAAE: nº344.0.045.000-10 e pela Comissão de Avaliação de Projetos de Pesquisa (CAPP/HU) do Hospital Universitário-UFPI. O estudo seguiu os critérios éticos, conforme as recomendações da Resolução 466/12 do Conselho Nacional de Saúde (CNS)9. Os participantes assinaram o Termo de Consentimento Livre e Esclarecido descritivo da pesquisa, garantindo-lhes o anonimato e assegurando o direito de desistirem da pesquisa a qualquer momento.

\section{Análise Estatística}

Os dados foram coletados, tabulados e processados pelo programa SPSS para Windows® versão 18.0 (SPSS Inc., 2000). Na análise estatística determinou a frequência, a média, o desvio padrão, correlação de Pearson e dendrogama.

\section{Resultados e Discussão}

Quanto às características do grupo caso, na análise dos dados pode-se observar que entre os pacientes com doença de Crohn $66,7 \%$ destes eram mulheres, $72,2 \%$ eram não-fumantes, $22,2 \%$ eram ex-fumantes e $83,3 \%$ relataram não fazer uso de álcool. Enquanto no grupo controle, 58,3\% da população estudada eram do sexo masculino, 77,8\% relataram ser ex-fumantes e 94,1\% faziam uso de álcool. Para o nível sócio-econômico, ambos os grupos apresentaram renda per capita semelhantes para todas as faixas salariais, variando de 300 a 3000 reais, sendo que o grupo caso apresentou maior escolaridade, pois $70 \%$ dos pacientes concluíram o ensino médio contra $30 \%$ do grupo controle.

Os valores médios e desvios padrão dos níveis séricos de PCRus, IL-10, IFN- $\gamma$, IL-8, TNF- $\alpha$ e cálculo do IADC dos pacientes com doença de Crohn e grupo controle estão apresentados na (Tabela 1). Destaca-se que todas as variáveis bioquímicas analisadas foram maiores nos pacientes com DC quando comparados ao grupo controle. Além disso, foi encontrada diferença significativa em relação à variável níveis séricos de PCRus, entre os grupos estudados ( $<<0,05)$. 
Tabela 1. Valores médios e desvios padrão dos níveis séricos de PCRus, IL-10, IFN- $\gamma$, IL-8, TNF- $\alpha$ para grupo caso e controle, e cálculo do IADC dos pacientes com doença de Crohn. Teresina, 2016.

\begin{tabular}{|c|c|c|c|c|c|}
\hline & & Crohn & Controle & & $\mathbf{p}$ \\
\hline Níveis séricos de PCRus (pg/mL) & & $8,06 \pm 9,25$ & $1,51 \pm 1,30$ & $5^{*}$ & 0,00 \\
\hline Níveis séricos de IL-10 (pg/mL) & 1261,55 & $484,91 \quad \pm$ & $0,059 \pm 0,07$ & & 0,11 \\
\hline Níveis séricos de IFN- $\gamma(\mathrm{pg} / \mathrm{mL})$ & & $1,85 \pm 6,62$ & $0,015 \pm 0,01$ & & 0,25 \\
\hline Níveis séricos de IL-8 (pg/mL) & & $0,045 \pm 0,04$ & $0,016 \pm 0,25$ & & 0,13 \\
\hline Níveis séricos de TNF- $\alpha$ (pg/mL) & & $69,95 \pm 259,82$ & $0,019 \pm 0,01$ & & 0,26 \\
\hline IADC & 126,86 & 127,83 & - & & - \\
\hline
\end{tabular}

*Correlação de Pearson significante para p<0,05. Fonte: Dados dos autores.

Na Tabela 2 estão demonstrados os valores médios e desvios padrão para idade, pressão arterial sistólica (PAS), pressão arterial diastólica (PAD), índice de massa corpórea (IMC), colesterol total, HDL-colesterol, triglicerídeos, LDLcolesterol para os pacientes com doença de Crohn e grupo controle. Observou-se que a média de idade entre os grupos analisados foi de 43,55; a média para o IMC foi de 25,88 para o grupo controle e de 22,05 para grupo caso; além disso, a média para os valores do perfil lipídico foi de 164,34, colesterol total, 40,75, HDL-c e 100,91, LDL-c para o grupo controle e, 131,12, colesterol total, 40,85, HDL-c e 76,05, LDL-c para o grupo caso. Notou-se diferença significativa entre as variáveis PAS, PAD, IMC, colesterol total e LDL-colesterol $(\mathrm{p}<0,05)$.

Tabela 2. Valores médios e desvios padrão da idade, pressão arterial sistólica (PAS), pressão arterial diastólica (PAD), índice de massa corpórea (IMC), colesterol total, HDL-colesterol, triglicerídeos, LDL-colesterol dos grupos estudados. Teresina, 2016.

\begin{tabular}{l|ccc}
\hline & Controle & Crohn & p \\
\hline Idade & $43,55 \pm 10,07$ & $41,38 \pm 15,33$ & 0,61 \\
PAS $(\mathrm{mmHg})$ & $130,27 \pm 12,81$ & $110,31 \pm 32,70$ & 0,02 \\
PAD $(\mathrm{mmHg})$ & $83,77 \pm 8,56$ & $73,12 \pm 10,83$ & 0,00 \\
IMC $\left(\mathrm{Kg} / \mathrm{m}^{2}\right)$ & $25,88 \pm 3,27$ & $22,05 \pm 3,71$ & 0,00 \\
Colesterol total $(\mathrm{mg} / \mathrm{dL})$ & $164,34 \pm 35,51$ & $131,12 \pm 31,77$ & 0,00 \\
HDL-colesterol $(\mathrm{mg} / \mathrm{dL})$ & $40,75 \pm 8,23$ & $40,85 \pm 13,96$ & $7 *$ \\
Triglicerídeos $(\mathrm{mg} / \mathrm{dL})$ & $113,09 \pm 55,18$ & $95,65 \pm 43,88$ & 0,98 \\
LDL-colesterol $(\mathrm{mg} / \mathrm{dL})$ & $100,91 \pm 33,25$ & $76,05 \pm 28,41$ & 0,32 \\
\hline
\end{tabular}

*Correlação de Pearson significante para $\mathrm{p}<0,05$. Fonte: Dados dos autores.

Os resultados da análise da correlação linear simples de Pearson entre os níveis de PCRus e as concentrações séricas das citocinas IL-10, IFN- $\gamma$, IL-8, TNF- $\alpha$ encontram-se na (Tabela 3). Verifica-se que houve correlação entre os níveis de PCRus e a concentração de IL-10 e IL-8 no grupo controle $(p<0,05)$. 
Tabela 3. Correlação entre PCRus e a concentração das citocinas IL-10, IFN- $\gamma$, IL-8, TNF- $\alpha$. Teresina, 2016.

\begin{tabular}{|c|c|c|c|c|}
\hline Grupos & IL-10 & IL-8 & IFN- $\gamma$ & TNF- $\alpha$ \\
\hline $\begin{array}{l}\text { Controle } \\
\text { PCR }\end{array}$ & $0,001 *$ & $0,024 *$ & 0,958 & 0,564 \\
\hline $\begin{array}{l}\text { Crohn } \\
\text { PCR }\end{array}$ & 0,786 & 0,052 & 0,606 & 0,601 \\
\hline
\end{tabular}

*Correlação de Pearson significante para $\mathrm{p}<0,05$. Fonte: Dados dos autores.

A análise da correlação de Pearson entre as citocinas nos dois grupos pode ser verificada na (Tabela 4). Observa-se correlação entre a IL-8 e a IL-10 no grupo controle. E, para o grupo com DC verifica-se correlação entre a IL-10 com IFN- $\gamma$ e com TNF- $\alpha(\mathrm{p}<0,05)$.

Tabela 4. Correlação entre as citocinas. Teresina, 2016.

\begin{tabular}{llcccc}
\hline & & IL-10 & IFN- $\gamma$ & IL-8 & TNF- $\alpha$ \\
\hline \multirow{3}{*}{ Controle } & IL-10 & - & 0,804 & 0,000 & 0,279 \\
& IFN- $\gamma$ & 0,804 & - & 0,756 & 0,175 \\
& IL-8 & 0,000 & 0,756 & - & 0,240 \\
& TNF- $\alpha$ & 0,279 & 0,175 & 0,240 & 0,000 \\
\hline \multirow{2}{*}{ Crohn } & IL-10 & - & 0,000 & 0,856 & 0,000 \\
& IFN- $\gamma$ & 0,000 & - & 0,691 & 0,746 \\
& IL-8 & 0,856 & 0,691 & 0,746 & - \\
\hline
\end{tabular}

*Correlação de Pearson significante para p<0,05. Fonte: Dados dos autores.

Na Figura 1 observa-se o dendograma de similaridade entre as dosagens de PCRus e das diferentes citocinas para a resposta inflamatória, onde é demonstrado uma similaridade entre as citocinas e que, a similaridade é maior entre a TFN- $\alpha$ e IFN- $\gamma$ com relação à PCRus, quando comparadas com IL-10 e IL-8 com PCRus.

Figura 1. Dendrograma de similaridade entre os níveis de citocinas e os níveis de PCRus.

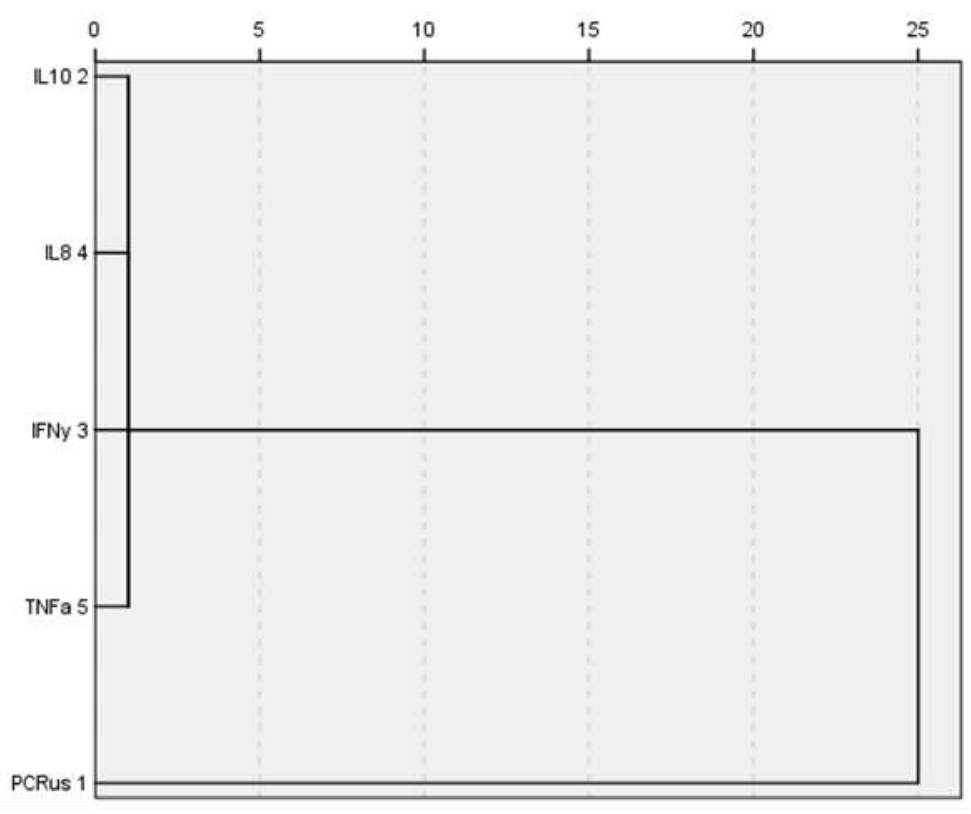

Fonte: Dados dos autores. 
Neste estudo foram avaliados os níveis de PCR-us e citocinas pró-inflamatórias, bem como foi investigada a relação entre estes sinalizadores inflamatórios e o risco de doenças cardiovasculares em pacientes com doença de Crohn. Dessa maneira, pôde-se verificar que os pacientes com doença de Crohn apresentaram níveis de PCR-us elevados (Tabela 1), sendo observada diferença significativa quando comparado ao grupo controle. Estes resultados corroboram os achados de Zorzi et al. (2013), Karatzas et al. (2014) e Pierote et al. (2018), que também identificaram concentrações plasmáticas de PCR-us elevadas em pacientes com doença de Crohn.

É oportuno mencionar que, a PCR-us é uma proteína de fase aguda (Castro; Viana, 2018) cuja concentração plasmática aumenta em $25 \%$ ou mais em resposta à inflamação, correspondendo ao mais sensível e precoce indicador de processos inflamatórios resultantes de infecções, traumas, alterações metabólicas e imunológicas, carcinomas, necrose tecidual ou outra desordem orgânica (Guimaraes et al., 2014; Pitthan; Martins, 2010). Embora não exista nenhum biomarcador não invasivo ideal para predizer a DC, a determinação da PCR-us é frequentemente utilizada durante a construção do diagnóstico dessa doença. De fato, os níveis séricos de PCR-us têm demonstrado valor prognóstico em indicar a efetividade da resposta à terapia e a atividade da doença de Crohn (Kellermayer et al., 2012).

No que diz respeito aos níveis séricos das citocinas IFN- $\gamma$, IL-10, TNF- $\alpha$ e IL-8 (Tabela 1), estas apresentaram valores mais elevados no grupo caso, apesar de não haver diferença significativa entre elas em relação ao grupo controle. Destaca-se o fato de que os níveis de IL-10 e TNF- $\alpha$ foram acentuadamente maiores nos pacientes com a doença. Esse resultado ressalta a associação bem estabelecida entre a doença de Crohn e uma resposta imune mediada por linfócitos $\mathrm{T}$ helper 1, que segregam citocinas como o TNF- $\alpha$. O TNF- $\alpha$, por sua vez, é um mediador pró-inflamatório chave perante a resposta da mucosa intestinal a antígenos, promovendo o recrutamento e estimulação de células inflamatórias (Müzes et al., 2012; Parker et al., 2019).

Nesse sentido, estudos de Gombošová et al. (2011) e Toptygina et al. (2014) verificaram resultados semelhantes em pacientes com DC, no qual foi possível observar níveis de TNF- $\alpha$ elevados em comparação com controles normais, indicando a sinalização pró-inflamatória mediada pelo TNF- $\alpha$. É importante mencionar que esta citocina pode ser usada como um marcador de atividade da doença, e fator discriminatório entre os dois tipos de DII (Ferraz et al., 2016; Müzes et al., 2012).

No que se refere à concentração de IL-10, esta apresentou valor elevado nos pacientes com doença de Crohn. A IL-10 é uma citocina pluripotente que desempenha um papel fundamental na regulação de respostas imunes e inflamatórias (Wilasco et al., 2017). Assim, ao manter eventos pró-inflamatórias sob controle, a IL-10 protege contra respostas imunitárias excessivas e danos aos tecidos. Portanto, mutações ocorridas nessa citocina ou em componentes da sua via de sinalização que reduzam ou suprimam as suas propriedades anti-inflamatórias foram relacionadas com a patogênese de doenças inflamatórias, como a doença de Crohn (Glocker et al., 2010; Wilasco et al., 2017).

Uma possível justificativa para a obtenção do resultado dessa pesquisa pode estar relacionada ao provável mecanismo de retroalimentação negativa, que visa à regulação da resposta imune no local da lesão a fim de recuperar o tecido afetado, visto que um estudo com população semelhante observou queda na produção de IL-10 no início do processo inflamatório, porém com o agravo da inflamação verificou-se aumento na produção de IL-10 (Gomes et al., 2014).

Resultados semelhantes foram observados por Wang et al. (2011) e Nunberg et al. (2018), que também verificaram que pacientes com DC apresentavam níveis significativamente mais elevados de IL-10 em comparação com controles saudáveis. Por ser uma citocina anti-inflamatória espera-se que níveis séricos elevados de IL-10, sejam favoráveis aos pacientes com doença inflamatória. Com efeito, concentrações reduzidas de IL-10 foram associadas com o aumento da gravidade da DC em comparação com altos níveis desta citocina (Marlow; Gent; Ferguson, 2013).

Sobre o IADC (Tabela 1), observou-se nesse estudo que os pacientes se apresentavam na fase de remissão da doença (IADC <150). Nesse aspecto, o estudo de Back et al. (2014) ao avaliar 54 pacientes com doença de Crohn, também encontrou remissão clínica da doença, com correlação significativa entre atividade ou remissão da doença e parâmetros antropométricos e 
composição corporal, porém não foi observado correlação entre o grau de atividade da doença e elevação da PCR.

Um aspecto importante a ser destacado, diz respeito à pressão diastólica e sistólica, colesterol total, LDL, triglicerídeos e IMC, que se apresentaram dentro dos valores de normalidade para os pacientes com DC, porém mais elevados no grupo controle (Tabela 2). Estes resultados foram semelhantes ao encontrado no estudo de Alkan et al. (2014), ao avaliar o risco de aterosclerose em pacientes com doença de Crohn, no qual observou-se que os valores de colesterol total, LDL, HDL e triglicerídeos, bem como o IMC encontravam-se dentro da normalidade.

No que diz respeito ao risco de doenças cardiovasculares, evidências sugerem que em pacientes com curso da doença de Crohn em atividade estão expostos a elevado risco para eventos ateroscleróticos, devido principalmente a um estado de inflamação seguida de hipercoagulabilidade. Além disso, alterações vasculares são comuns nesses pacientes, visto que modificações precoces na parede arterial têm sido observadas, incluindo disfunção endotelial e aumento da espessura da camada íntima-média, associados com alterações do perfil lipídico apoiam o risco aumentando de doença aterogênica na DC (Andersen; Jess, 2014; Thapa, 2015).

De fato, o estado inflamatório envolve diversos mecanismos não específicos, incluindo liberação de citocinas e outros mediadores inflamatórios (como TNF- $\alpha$, IL-1, e PCR), o que pode contribuir para a alteração do equilíbrio hemostático para um estado trombótico. Somam-se a esse estado os processos fisiopatológicos ocorridos na parede do cólon em pacientes com DC, os quais compartilham muitas características com as alterações na parede arterial durante a progressão da aterosclerose e, finalmente, a ruptura da placa aterosclerótica e trombose (Aguilar-Tablada et al., 2016; Kristensen et al., 2013).

Desse modo, níveis aumentados de PCR-us e LDL-c associados a concentrações reduzidas de HDL-c agravam o risco de evento cardiovascular, independente dos fatores de risco como idade, cigarro, obesidade, pressão arterial e diabetes. A inflamação tem um papel importante na aterosclerose, acelerando esse processo e levando a uma ruptura da placa aterosclerótica. Evidências clínicas demonstram que alterações nos níveis de PCR-us estão associadas ao risco de mortalidade, em curto e longo prazo, em pacientes com doença isquêmica cardíaca aguda e crônica, e também naqueles com risco de desenvolver aterosclerose (Ribas et al., 2015).

No entanto, considerando os achados dessa pesquisa não foi possível fazer essa relação, visto que os valores de LDLc, HDL-c, colesterol total e triglicerídeos estavam dentro da normalidade para os pacientes com doença de Crohn. Porém sobre aspecto, é possível afirmar que a elevação dos níveis de PCRus está associado ao processo inflamatório crônico, e que parece ser um preditor de aumento do risco cardiovascular.

Ao ser determinada a correlação de Pearson (Tabela 3) entre PCRus e as citocinas observou-se que houve correlação entre as PCRus com IL-10 e IL-8 no grupo controle. Conforme Baldaia et al. (2002), a ação de algumas citocinas próinflamatórias, como a IL-8, pode gerar estímulos para produção de proteínas de fase aguda, como a PCRus, expressão de moléculas de adesão, proliferação fibroblástica e citotoxicidade.

Para a correlação de Pearson entre as citocinas nos dois grupos, verificou-se a presença de correlação significante $(\mathrm{p}<0,05)$ entre a IL-8 e a IL-10 no grupo controle. Entretanto, para o grupo caso notou-se que houve correlação entre a IL-10, IFN- $\gamma$ e TNF- $\alpha$. Nesse sentido Tilg et al. (2002) e Wu et al. (2017) apontam que as concentrações plasmáticas de IFN- $\gamma$ encontram-se elevadas em pacientes com doença de Crohn na sua fase ativa e correlacionam-se com a atividade clínica da doença, bem com o TNF- $\alpha$, visto que, este é um mediador central na ativação da cascata inflamatória na DC (Müzes et al., 2012; Li et al., 2017).

Com o dendograma (Figura 1), pôde-se observar similaridade entre as citocinas e que, há maior similaridade entre TFN- $\alpha$ e IFN- $\gamma$ com relação às PCRus, quando comparadas com IL-10 e IL-8 com PCRus. 


\section{Conclusão}

Diante dos dados obtidos, os quais confirmaram níveis de PCRus mais elevados no grupo caso sugere-se que os pacientes com doença de Crohn, apresentam risco cardiovascular aumentado quando comparados com pessoas sem a doença e com outros fatores de risco. Conforme os estudos apontam, o risco cardiovascular é aumentado quando associado a outros fatores como: circunferência da cintura, IMC e frequência de certas morbidades.

Logo, na doença de Crohn, acentua-se a relevância do teste de rotina para esses marcadores, cuja eficácia na previsão de eventos cardiovasculares não deve ser ignorada no acompanhamento dos pacientes com doenças inflamatórias crônicas. Além da PCRus ficou demonstrado neste estudo que as citocinas estão mais elevadas nesses pacientes, dessa forma pode-se sugerir que esta doença está diretamente relacionada com a liberação de citocinas inflamatórias.

Nesse sentido, faz-se importante estudos futuros que esclareçam ainda mais os mecanismos pelos quais as citocinas inflamatórias se relacionam com o risco cardiovascular em pacientes com doença de Crohn.

\section{Agradecimentos}

Agradecemos a professora e orientadora Regina Célia de Assis (in memorian) pela possibilidade de realização desse trabalho sob sua orientação e pelo auxilio na construção desse artigo. Professora Regina apesar de não está mais entre nós deixou muitos ensinamentos e exemplos.

\section{Referências}

Aguilar-Tablada, C. T., Navarro-Alarcón, M., Quesada Granados, J., Samaniego Sánchez, C., Rufián-Henares, J. Á., \& Nogueras-Lopez, F. (2016). Ulcerative colitis and Crohn's disease are associated with decreased serum selenium concentrations and increased cardiovascular risk. Nutrients, 8(12), 780.

Alkan, E., Karakaş, M. S., \& Yıldırım, B. (2014). Evaluation of increased subclinical atherosclerosis risk with carotid intima-media thickness and pulse wave velocity in inflamatory bowel disease. Turk J Gastroenterol, 25(1), 20-25.

Andersen, N. N., \& Jess, T. (2014). Risk of cardiovascular disease in inflammatory bowel disease. World journal of gastrointestinal pathophysiology, 5(3), 359.

Back, I. R. (2014). Influência do estado nutricional e da composição corporal na evolução clínica dos pacientes com doença inflamatória intestinal.

Baldaia, C., Portela, F., Andrade, P., Leitão, M. C., \& Freitas, D. (2002) Doença de Crohn: TNF- $\gamma$ e outras citocinas. J. Port. Gastrenterol, 9: $187-195$.

Barros, A. (2015). Composição corporal, depressão, qualidade de vida e mortalidade em hemodiálise.

Brasil. Portaria SAS/MS nº 858, de 04 de novembro de 2002. Aprova o Protocolo Clínico e Diretrizes Terapêuticas-Doença de Crohn, Brasília, 2002.

Brasil. Resolução n466/2012. Conselho Nacional de Pesquisa com Seres Humanos. Brasília, 2012.

Castro, K. A., \& Viana, E. D. S. M. (2018). Avaliação da proteína C reativa ultrassensível em ratos diabéticos tratados com resveratrol. Revista Eletrônica Científica Da UERGS, 4(1), 03-16.

Ferraz, F. B. (2016). Panorama geral sobre doenças inflamatórias intestinais: Imunidade e suscetibilidade da doença de crohn e colite ulcerativa. Journal of Health Sciences, 18(2), 139-143.

Ferreira, A. A., Natali, M. R. M., de Oliveira Delani, T. C., Martins, R. M., \& Prestes, T. S. (2010). Papel do sistema imune e atuação dos probióticos na doença de Crohn. Arquivos de Ciências da Saúde da UNIPAR, 14(2).

Glocker, E. O., Kotlarz, D., Klein, C., Shah, N., \& Grimbacher, B. (2011). IL-10 and IL-10 receptor defects in humans. Annals of the New York Academy of Sciences, 1246(1), 102-107.

Gombošová, L., Lazúrová, I., Zakuciová, M., Čurová, K., Kmet’ová, M., Petrášová, D., \& Siegfried, L. (2011). Genes of intestinal Escherichia coli and their relation to the inflammatory activity in patients with ulcerative colitis and Crohn's disease. Folia microbiologica, $56(5), 367$.

Gomes, R. G. (2014). Associação de polimorfismos nas sequências regulatórias dos genes HLA-G, IL-10 e TNF e a sua respectiva expressão gênica em lesões de mucosa em pacientes portadores de doença inflamatória intestinal (Doctoral dissertation, Centro de Pesquisas Aggeu Magalhães).

Guimarães, A.O., de Brito, A. M. G., Goes, C. S., de Araújo Reis, A., \& Reges, A. L. S. (2014). Proteína C reativa e velocidade de hemossedimentação na avaliação laboratorial de processos inflamatórios. Scire Salutis, 4(1), 6-16. 
Kalogeropoulos, A. P., Tang, W. W., Hsu, A., Felker, G. M., Hernandez, A. F., Troughton, R. W., \& Butler, J. (2014). High-sensitivity C-reactive protein in acute heart failure: insights from the ASCEND-HF trial. Journal of cardiac failure, 20(5), 319-326.

Karatzas, P. S., Mantzaris, G. J., Safioleas, M., \& Gazouli, M. (2014). DNA methylation profile of genes involved in inflammation and autoimmunity in inflammatory bowel disease. Medicine, $93(28)$.

Kellermayer, R., Mir, S. A., Nagy-Szakal, D., Cox, S. B., Dowd, S. E., Kaplan, J. L., \& Winter, H. S. (2012). Microbiota separation and C-reactive protein elevation in treatment naïve pediatric granulomatous Crohn disease. Journal of pediatric gastroenterology and nutrition, 55(3).

Koenig, W. (2013). High-sensitivity C-reactive protein and atherosclerotic disease: from improved risk prediction to risk-guided therapy. International journal of cardiology, 168(6), 5126-5134.

Kristensen, S. L., Ahlehoff, O., Lindhardsen, J., Erichsen, R., Jensen, G. V., Torp-Pedersen, C., \& Hansen, P. R. (2013). Disease activity in inflammatory bowel disease is associated with increased risk of myocardial infarction, stroke and cardiovascular death-a Danish nationwide cohort study. PloS one, 8(2), e56944.

Li, J., Doty, A. L., Tang, Y., Berrie, D., Iqbal, A., Tan, S. A., \& Glover, S. C. (2017). Enrichment of IL-17A+ IFN- $\gamma+$ and IL-22+ IFN- $\gamma+$ T cell subsets is associated with reduction of NKp44+ ILC3s in the terminal ileum of Crohn's disease patients. Clinical \& Experimental Immunology, $190(1)$, $143-153$.

Marlow, G. J., van Gent, D., \& Ferguson, L. R. (2013). Why interleukin-10 supplementation does not work in Crohn's disease patients. World journal of gastroenterology: WJG, 19(25), 3931.

Müzes, G., Molnár, B., Tulassay, Z., \& Sipos, F. (2012). Changes of the cytokine profile in inflammatory bowel diseases. World journal of gastroenterology: WJG, 18(41), 5848

Nunberg, M. Y., Werner, L., Kopylov, U., Haberman, Y., Lahad, A., Weiss, B., \& Shouval, D. S. (2018). Impaired IL-10 receptor-mediated suppression in monocyte from patients with Crohn disease. Journal of pediatric gastroenterology and nutrition, 66(5), 779-784.

Papacosta, N. G., Nunes, G. M., Pacheco, R. J., Cardoso, M. V., \& Guedes, V. R. (2017). Doença de crohn. Revista de Patologia do Tocantins, 4(2), 25-35.

Parker, A., Vaux, L., Patterson, A. M., Modasia, A., Muraro, D., Fletcher, A. G., \& Pin, C. (2019). Elevated apoptosis impairs epithelial cell turnover and shortens villi in TNF-driven intestinal inflammation. Cell death \& disease, 10(2), 1-13.

Pierote, N. R., Braz, A. F., Barros, S. L., Neto, J. M. M., Parente, J. M. L., Maria da Cruz, M. S., \& do Noscimento Nogueira, N. (2018). Effect of mineral status and glucocorticoid use on bone mineral density in patients with Crohn's disease. Nutrition, 48, 13-17.

Pithan, E., \& OMO, M. (2010). Marcadores inflamatórios e de disfunção endotelial: qual o papel na predição de risco. Rev Soc Cardiol RS, 18(20), 1-11.

Onali, S., Calabrese, E., Petruzziello, C., Zorzi, F., Sica, G., Fiori, R., \& Biancone, L. (2012). Small intestine contrast ultrasonography vs computed tomography enteroclysis for assessing ileal Crohn's disease. World journal of gastroenterology: WJG, 18(42), 6088.

Ribas, J. T., Belló, C., Ito, C. A. S., Mine, J. C., \& Vellosa, J. C. R. (2015). Alterações metabólicas e inflamatórias na gestação. Revista de Ciências Farmacêuticas Básica e Aplicada, 36(2).

Sercelik, A., Tanrıverdi, O., Askin, L., \& Turkmen, S. (2021). A associação da relação proteína C-reativa/albumina em pacientes com ectasia da artéria coronária isolada. Arquivos Brasileiros de Cardiologia, 116(1), 48-54.

Soares, J. A., da Silveira Schauren, J., \& Stroparo, E. (2018). Doença de crohn: revisão de literatura. Revista Eletrônica Biociências, Biotecnologia E Saúde, $11(21), 78-83$.

Thapa, S. D., Hadid, H., Imam, W., Schairer, J., \& Jafri, S. M. (2015). Effect of Inflammatory Bowel Disease-Related Characteristics and Treatment Interventions on Cardiovascular Disease Incidence. The American journal of the medical sciences, 350(3), $175-180$.

Tilg, H., Van Montfrans, C., Van den Ende, A., Kaser, A., Van Deventer, S. J. H., Schreiber, S., \& Weiss, G. (2002). Treatment of Crohn's disease with recombinant human interleukin 10 induces the proinflammatory cytokine interferon $\gamma$. Gut, 50(2), 191-195.

Toptygina, A. P., Semikina, E. L., Bobyleva, G. V., Miroshkina, L. V., \& Petrichuk, S. V. (2014). Cytokine profile in children with inflammatory bowel disease. Biochemistry (Moscow), 79(12), 1371-1375.

Torres, J. A. D. P., Santana, R. M. D., Torres, F. A. D. P., Moura, A. R., \& Torres Neto, J. D. R. (2011). Doenças inflamatórias intestinais no Hospital Universitário da Universidade Federal de Sergipe: manifestações extraintestinais. Revista Brasileira de Coloproctologia, 31(2), 115-119.

Valadas, J., Foigt, V., de Aguiar Raupp, W., de Freitas, T. A. B., \& Rotta, L. (2019). Correlação entre os resultados de proteína c-reativa e velocidade de hemossedimentação. Revista de Atenção à Saúde, 17(60).

Vilela, E. G., da Gama Torres, H. O., Martins, F. P., de Abreu Ferrari, M. D. L., Andrade, M. M., \& da Cunha, A. S. (2012). Evaluation of inflammatory activity in Crohn's disease and ulcerative colitis. World journal of gastroenterology: WJG, 18(9), 872.

Wang, A. H., Lam, W. J., Han, D. Y., Ding, Y., Hu, R., Fraser, A. G., ... \& Morgan, A. R. (2011). The effect of IL-10 genetic variation and interleukin 10 serum levels on Crohn's disease susceptibility in a New Zealand population. Human immunology, 72(5), 431-435.

Wilasco, M. I. A., Uribe-Cruz, C., Santetti, D., Fries, G. R., Dornelles, C. T. L., Silveira, T. R. (2017). IL-6, TNF- $\alpha$, IL-10, and nutritional status in pediatric patients with biliary atresia. Jornal de Pediatria (Versão em Português), 93(5), 517-524.

Wu, H., Wang, L., Zhang, D., Qian, J., Yan, L., Tang, Q., \& Zou, X. (2017). PRDM5 promotes the apoptosis of epithelial cells induced by IFN- $\gamma$ during Crohn's disease. Pathology-Research and Practice, 213(6), 666-673. 
Research, Society and Development, v. 10, n. 4, e17810414088, 2021

(CC BY 4.0) | ISSN 2525-3409 | DOI: http://dx.doi.org/10.33448/rsd-v10i4.14088

Yuan, C., Chen, W. X., Zhu, J. S., Chen, N. W., Lu, Y. M., Ou, Y. X., \& Chen, H. Q. (2013). IL-10 treatment is associated with prohibitin expression in the Crohn's disease intestinal fibrosis mouse model. Mediators of Inflammation, 2013.

Zorzi, F., Monteleone, I., Sarra, M., Calabrese, E., Marafini, I., Cretella, M., \& Monteleone, G. (2013). Distinct profiles of effector cytokines mark the different phases of Crohn's disease. PLoS One, 8(1), e54562. 